\title{
IL-25 stimulates M2 macrophage polarization and thereby promotes mitochondrial respiratory capacity and lipolysis in adipose tissues against obesity
}

\author{
Juan Feng ${ }^{1,7}$, Lingyi Li ${ }^{1,7}$, Zhiying Ou ${ }^{2,7}$, Qiao $\mathrm{Li}^{1}$, Baoyong Gong ${ }^{3}$, Zhenxian Zhao ${ }^{4}$, Weiwei Qi ${ }^{1}$,
} Ti Zhou ${ }^{1}$, Jun Zhong1, Weibin Cai ${ }^{5}$, Xia Yang ${ }^{1}$, Aiping Zhao ${ }^{6}$, Guoquan Gao ${ }^{1}$ and Zhonghan Yang ${ }^{1}$

Obesity and associated metabolic diseases are characterized by a chronic low-grade inflammatory state with the infiltration of many inflammatory cells, especially macrophages. Immune molecules, including some cytokines, have a close relationship with metabolism. Interleukin (IL)-25 is a member of the IL-17 cytokine family that can regulate macrophages and alleviate some metabolic dysfunction; however, its role and mechanisms in lipid metabolism remain to be extensively clarified. Human serum and liver biopsy specimens, high-fat diet-induced obesity mice and DB/DB (Lepr-/-) animal models were used to examine IL-25 expression in obesity and nonalcoholic fatty liver diseases (NAFLD). To observe the role of IL-25 in lipid metabolism, model mice were administered with IL-25 or adoptively transferred with IL-25-educated macrophages in vivo, whereas bone marrow-derived macrophages, the macrophage cell line RAW264.7 and adipocytes differentiated from 3T3-L1 were used in vitro. IL-25 was decreased in NAFLD patients and obese mice. In addition, IL-25 reduced body weight gain and lipid accumulation, enhanced lipid uptake by macrophages and increased the expression of lipolysis and $\beta$-oxidation enzymes via alternatively activating macrophages. IL-25 also promoted lipolysis and suppressed lipogenesis in adipocytes co-cultured with the IL-25-educated macrophages. Furthermore, IL-25 improved the mitochondrial respiratory capacity and oxygen consumption rate of macrophages and produced more NAD+/NADH and ATP. In conclusion, IL-25 can stimulate M2 macrophage polarization and thereby promote lipolysis and mitochondrial respiratory capacity, highlighting the potential for IL-25 to be used as a therapeutic agent against obesity and associated metabolic syndromes. Cellular \& Molecular Immunology (2018) 15, 493-505; doi:10.1038/cmi.2016.71; published online 13 February 2017

Keywords: adipocyte; immunometabolism; IL-25; macrophage; obesity

\section{INTRODUCTION}

Obesity is an emerging public health problem worldwide. According to the World Health Organization, more than $39 \%$ of adults are overweight, and $\sim 13 \%$ were clinically obese in 2014..$^{1,2}$ Obesity is related to many diseases, such as nonalcoholic fatty liver disease (NAFLD), insulin resistance, cardiovascular diseases and some endocrine diseases. ${ }^{3-5}$ Obesity is a risk factor not only for metabolic diseases but also for pulmonary diseases and cancer. ${ }^{6,7}$ Therefore, it is urgent to seek appropriate therapeutic and preventive measures for obesity and its complications.

Metabolic diseases, such as obesity and diabetes, are well known to be related to overnutrition and a sedentary lifestyle, and they are also regarded as an immune imbalance between type- 1 and type- 2 immunity because these metabolic diseases are usually characterized by chronic low-grade systemic inflammation. Inflammatory factors, including cytokines, and mediators may be the causative factors of many metabolic

\footnotetext{
${ }^{1}$ Program of Molecular Medicine, Affiliated Guangzhou Women and Children's Hospital, Zhongshan School of Medicine, Sun Yat-sen University, Guangzhou 510080, China; ${ }^{2}$ Institute of Pediatrics, Affiliated Guangzhou Women and Children's Medical Center, Sun Yat-sen University, Guangzhou 510623, China; ${ }^{3}$ Guangdong Provincial Key Laboratory of Laboratory Animals, Guangdong Laboratory Animals Monitoring Institute, Guangzhou 510063, China; ${ }^{4}$ PancreatoBiliary Surgery, First Affiliated Hospital, Sun Yat-sen University, Guangzhou 510080, China; ${ }^{5}$ Guangdong Engineering and Technology Research Center for Disease-Model Animals, SUN Yat-sen University, Guangzhou 510006, China and ${ }^{6}$ Department of Radiation Oncology, Department of Medicine, University of Maryland School of Medicine, Baltimore, MD 21201, USA

${ }^{7}$ These authors contributed equally to this work.

Correspondence: Dr Z Yang, PhD or Professor G Gao, PhD, Department of Biochemistry, Zhongshan School of Medicine, Sun Yat-sen University, 74 Zhongshan 2nd, Guangzhou 510080 GD, China.

E-mail: yangzhh@mail.sysu.edu.cn or gaogq@mail.sysu.edu.cn

Received: 15 July 2016; Revised: 13 November 2016; Accepted: 14 November 2016
} 
disorders, such as diabetes, obesity and insulin resistance. ${ }^{8}$ Adipose tissue has long been considered an energy-storage organ, whereas it is increasingly accepted that adipose tissue is an endocrine organ that can secrete several types of hormones and cytokines. ${ }^{9-11}$ Immune cells, such as macrophages, in adipose tissue are important to maintain the adipose tissue homeostasis and regulate body weight. ${ }^{12}$ Typically, accumulated macrophages in adipose tissue are closely associated with systemic low-grade inflammation, obesity-linked liver damage and insulin resistance. ${ }^{13}$ Macrophages are mainly classified into two phenotypes, namely classically activated macrophages (M1), which predominantly produce inflammatory factors, and alternatively activated macrophages (M2), which mainly generate anti-inflammatory factors. Macrophage accumulation in adipose tissue is increased in obese people and animals, and obesity can promote the accumulation of pro-inflammatory M1 macrophages in adipose tissue. ${ }^{12,14}$

Adipocytes, especially adipose tissue-derived stem cells from obese but not lean subjects promote Th17 differentiation and activation, ${ }^{15}$ and, in turn, interleukin (IL)-17 can inhibit adipogenesis. ${ }^{16}$ IL-25, also called IL-17E, is a member of the IL-17 family, which includes IL-17A to IL-17F. ${ }^{17-20}$ IL-25 participates in Th2-type anti-inflammatory responses, which are associated with some immune diseases, such as asthma and allergic inflammation. ${ }^{18,20}$ IL-25 is also considered to be a potential therapeutic agent for hepatic steatosis and fulminant hepatitis. $^{21,22}$ Compared with wild-type mice, enlarged livers and increased body weight were observed in IL-25 knockout mice. $^{21}$ IL-25 can reduce body weight and improve glucose tolerance with the increased infiltration of innate lymphoid type- 2 cells and type I and type II NKT cells in visceral adipose tissue. ${ }^{23}$ However, the role and mechanism of IL-25- and IL-25-stimulated macrophages in fat metabolism remain to be elucidated. In the present study, we examined the expression level of IL-25 in metabolic diseases, investigated the role of IL-25 in macrophage polarization and consequent lipid metabolism, elucidated the metabolic mechanism of IL-25-triggered lipolysis and provided evidence of IL-25 as a factor in immunometabolism against obesity.

\section{MATERIALS AND METHODS}

\section{Ethics statement}

All the animal experiments were conducted with the approval of the Animal Care and Use Committee of Sun Yat-sen University (Approval ID: SCXK2011-0029). The clinical investigation complied with the ethical principles established by the 1964 Declaration of Helsinki. The sample collection from patients was approved by the Ethics Committee of the Guangzhou Women and Children's Hospital and the First Affiliated Hospital of Sun Yat-sen University. All the patients or family members involved provided written informed consent.

\footnotetext{
Animals

C57BL/6 wild-type mice were purchased from the Laboratory Animal Center of Sun Yat-sen University (Guangzhou, China). The care, use and treatment of all animals in this study were in
}

strict accordance with the principles set forth by the Animal Care and Use Committees of Sun Yat-sen University. For these experiments, 4- to 5-week-old male mice were fed a normal chow diet (NCD) or a high-fat diet (HFD, 60\% of calories, D12492, Research Diets Inc., NJ, USA) for 3 months to create the obesity model, and body weight and food consumption were monitored. At the end of 3 months of the NCD or HFDinduction program, mice were intraperitoneally injected with bovine serum albumin (BSA) or IL-25 (1 $\mu \mathrm{g}$ in $100 \mu \mathrm{l}$ phosphate-buffered saline (PBS) for each mouse, Biolegend, San Diego, CA, USA) every day for 7 days followed by every other day for an additional 16 days for a total of 23 days. During the HFD treatment, the serum and tissues of mice were also collected at $0,1,2,4,12$ and 16 weeks to explore changes in the level of IL-25. DB/DB (Lepr - / - ) mice were purchased from the Nanjing Laboratory Animal Center (Nanjing, China).

\section{Macrophage culture, adoptive transfer}

Bone marrow-derived macrophages (BMMCs) were generated as previously described. ${ }^{24}$ Briefly, mononuclear cells were flushed from the femurs and tibias of C57BL/6 mice and cultured overnight. The non-adherent cells were collected and centrifuged at 1200 r.p.m. for $10 \mathrm{~min}$. The cells were then treated with Red Cell Lysis Buffer (Sigma-Aldrich, St Louis, MO, USA). Mature macrophages were obtained from the differentiated mononuclear cells and stimulated with $20 \mathrm{ng} / \mathrm{ml}$ macrophage colony-stimulating factor (Biolegend, \#576406) for 7 days. Mouse RAW264.7 macrophages were from the American Type Culture Collection (ATCC, Manassas, VA, USA). BMMCs and mouse RAW264.7 macrophages were cultured in dulbecco's modified eagle medium with $10 \%$ fetal bovine serum (Gibco by Life Technologies, Bleiswijk, the Netherlands) and 1\% penicillin/streptomycin. Cells were cultured at $37^{\circ} \mathrm{C}$ with $5 \% \mathrm{CO}_{2}$. For adoptive transfer, BMMCs stimulated with or without IL-25 $(50 \mathrm{ng} / \mathrm{ml}$, Biolegend, \#587306), IL-15 (25 ng/ml, Biolegend) and interferon gamma (IFN $\gamma ; 50 \mathrm{ng} / \mathrm{ml}$, Biolegend) for 2 days were collected in cold PBS, and $1 \times 10^{6}$ cells were adoptively transferred into every mouse intraperitoneally after a HFD for 3 months. The adoptive transfer was performed two times interspaced for a week, and all mice were killed for analysis 7 days after the second adoptive transfer experiment.

\section{Co-culture of macrophages and adipocytes}

Confluent 3T3-L1 (ATCC) cells seeded in six-well plates were induced into mature adipocytes with $0.5 \mathrm{mM}$ isobutylmethylxanthine (Sigma-Aldrich), $1 \mu \mathrm{M}$ dexamethasone (SigmaAldrich) and $10 \mu \mathrm{g} / \mathrm{ml}$ insulin (Sigma-Aldrich) in dulbecco's modified eagle medium containing $10 \%$ fetal bovine serum for 2 days and then treated with dulbecco's modified eagle medium containing $10 \mu \mathrm{g} / \mathrm{ml}$ insulin and $10 \%$ fetal bovine serum for 7 days. Then, $3 \times 10^{5}$ RAW264.7 cells/well were seeded into Transwell chambers (Corning, New York, NY, USA) on top of the mature adipocytes for 2 days. Different cytokines, such as IL-5 (50 ng/ml, Biolegend), IL-15 (25 ng/ml, Biolegend), IL-25 $(50 \mathrm{ng} / \mathrm{ml}$, Biolegend $), \quad$ IFN $\gamma \quad(50 \mathrm{ng} / \mathrm{ml}$, Biolegend $)$ and 
lipopolysaccharide (LPS; $10 \mathrm{ng} / \mathrm{ml}$, Sigma), were administered for treatment of the macrophages.

\section{RNA Extraction and Quantitative Real-Time PCR}

Total RNA was extracted from tissues or cells using TRIzol reagent (Invitrogen, Carlsbad, CA, USA). In total, $1000 \mathrm{ng}$ total RNAs were reverse-transcribed to complementary DNA (cDNA) using the Prime Script RT Reagent Kit Perfect Real-Time Kit (TaKaRa Bio Inc., Kusatsu, Shiga, Japan). The cDNA was then used for quantitative real-time PCR (qPCR) using SYBR Premix Ex Taq (TaKaRa Bio Inc.). ${ }^{25}$ The expression of the target mRNA was determined using the comparative cycle threshold method normalized to the $18 \mathrm{~S}$ gene as previously described. ${ }^{26}$ The primers used for the qPCR are listed in Supplementary Table 1.

\section{Oil Red O Staining}

The slices were fixed with $4 \%$ paraformaldehyde. After fixation, lipid droplets of tissue slices were stained with Oil Red O prepared in 2-propanol $(0.5 \%)$ as the stock solution, and the working resolution was freshly diluted with distilled deionized water. The slices were incubated with the Oil Red O working solution $(60 \mathrm{ml}$ stock solution mixed with $40 \mathrm{ml}$ deionized water) for $15 \mathrm{~min}$ and stained with hematoxylin for $30 \mathrm{~s}$. All the slices were observed under a light contrast microscope.

\section{Western blotting}

Cells were harvested and lysed for preparation. The protein concentration was determined using the KeyGen Protein Assay Kit (KeyGen, Nanjing, Jiangsu, China) according to the manufacturer's instructions. Equal amounts of protein extracts were subjected to a western blot analysis. The proteins transferred to the polyvinylidene fluoride membrane (Millipore, Boston, MA, USA) were probed with primary antibodies specific for adipose triglyceride lipase (ATGL; 1:1000; Cayman Chemical Co., Madison, MI, USA, \#10006409), p-ACC (1:1000; Cell Signaling Technology, Danvers, MA, USA, \#3661S), ACC (1:1000; CST, \#3676S), monoacylglycerol Lipase (MAGL) (1:1000; Cayman Chemical Co., \#100035), phosphorylated hormone sensitive lipase (p-HSL) (1:1000; Abcam, Cambridge, MA, USA, \#ab109400), hormone sensitive lipase (HSL) (1:1000; CST, \#18381S), CPT1 $\alpha$ (1:1000; Proteintech Group, Chicago, IL, USA, \#15184-1-AP) and $\beta$-actin (1:10000, Sigma-Aldrich, \#A5441) overnight at $4{ }^{\circ} \mathrm{C}$. The membranes were then washed with TBST and incubated with peroxidaseconjugated secondary antibodies at $4{ }^{\circ} \mathrm{C}$ for $3 \mathrm{~h}$. The following secondary antibodies were used: goat anti-rabbit IgG/HRP (1:1000; Vector Laboratories, Youngstown, OH, USA, \#PI1000) and goat anti-mouse IgG/HRP (1:1000; Vector Laboratories, Youngstown, OH, USA, \#PI2000). Chemiluminescence was developed using ECL western blotting substrate and quantified using the ImageJ software (NIH, Bethesda, MD, USA).

\section{ELISA of IL-25 in human liver biopsy specimens and mouse tissues}

Biopsies of liver sections and serum were acquired from a total of 14 patients. Six of these patients had hepatic steatosis, initially diagnosed with an ultrasound examination and later confirmed by a liver biopsy evaluation. The other eight patients had no hepatic steatosis and were used as controls. Their medical histories were reviewed retrospectively by a medical doctor that specializes in gastroenterology and hepatology. All the patients were $>18$ years old at the time of biopsy and had no history of alcohol abuse, serologic evidence of viral hepatitis, blood transfusions or history of other competing etiologies for hepatic steatosis and coexisting causes for chronic liver disease. $^{27}$ For the sample preparation of tissues, liver or epididymal white adipose tissues (eWAT) were homogenized in ice-cold PBS with a protease inhibitor cocktail, followed by centrifugation at $1500 \mathrm{~g}$ for $10 \mathrm{~min}$. Then, the supernatants were collected for the enzyme-linked immunosorbent assay (ELISA), and the protein concentration was measured using the KeyGen protein assay kit (KeyGen). The expression levels of IL-25 in the cell supernatant, serum and tissues were detected using the Mouse IL-25 ELISA Kit (Biolegend, \#434004) or the human IL-25 ELISA Kit (Cloud-Clone Corp., \#SEB694Hu) according to the manufacturer's instructions.

\section{Immunofluorescence staining}

For the immunofluorescence staining, epididymal fat and liver tissue sections were fixed in $4 \%$ paraformaldehyde and blocked with goat serum for $1 \mathrm{~h}$ at room temperature. The sections were then incubated with the F4/80 (1:200, Abcam, \#ab6640) and ARG1 (1:200, Santa Cruz Biotechnology, Santa Cruz, CA, USA, \#sc-20150) antibodies overnight at $4{ }^{\circ} \mathrm{C}$. After being washed, the slices were incubated with Alex Fluor 488-Donkey anti-rat IgG (H+L; 1:200, Life Technologies, \# A21208) and Alex Fluor 594-Donkey anti-rabbit IgG (1:200, Life Technologies, \#R37119) for $1 \mathrm{~h}$ at room temperature and then with DAPI (4',6-diamidino-2-phenylindole, Sigma-Aldrich, MO, USA) for $15 \mathrm{~min}$ at room temperature. The slices were digitally photographed with a confocal microscope.

\section{Measurement of triglycerides, free fatty acids, HDL, LDL/VLDL, ATP and $\mathrm{NAD}^{+} / \mathrm{NADH}$}

The triglycerides (TGs) and free fatty acids (FFAs) in the tissues and plasma were measured using the EnzyChrom Triglyceride Assay Kit (BioAssay Systems, Hayward, CA, USA, Cat\# ETGA-200) and EnzyChrom Free Fatty Acid Assay Kit (BioAssay Systems, Hayward, CA, USA, Cat\#EFFA-100) according to the manufacturer's instructions. In addition, the levels of high-density lipoprotein (HDL) and low-density lipoprotein/very low-density lipoprotein (LDL/VLDL) in the serum were also measured using the EnzyChrom AF HDL) and (LDL /VLDL) Assay Kit (BioAssay Systems, Hayward, CA, USA, E2HL-100) according to the manufacturer's instructions. The ATP and $\mathrm{NAD}^{+} / \mathrm{NADH}$ produced by RAW264.7 macrophages or BMMC were measured with the ATP detection kit (Beyotime Biotechnology, Beyotime Institute of Biotechnology, Haimen, Jiangsu, China, \#S0026) and $\mathrm{NAD}^{+} / \mathrm{NADH}$ Quantification Colorimetric Kit (Biovision, San Francisco, CA, USA, \#K337-100) according to the manufacturer's instructions. 


\section{MitoTracker staining}

BMMC and RAW264.7 macrophages were incubated with 100 nM MitoTracker Green FM (Life Technologies, \#M7514) for $30 \mathrm{~min}$ according to the manufacturer's instructions. Then, the cells were washed with PBS and visualized under a confocal microscope.

\section{Adipocyte area quantification}

eWAT sections were hematoxylin and eosin-stained and imaged at $\times 40$ magnification. The adipocyte size was analyzed using the ImageJ software via drawing ellipses circumscribing white adipocytes. Two to three images and 15-20 adipocytes per image were measured. ${ }^{28}$

\section{Measurement of oxygen consumption rate}

The oxygen consumption rate was measured with a Seahorse Bioscience XF-96 extracellular flux analyzer (Seahorse Bioscience) according to the manufacturer's instructions as detailed elsewhere. ${ }^{29-31}$ Cells stimulated with different cytokines were seeded in XF 24-well plate (Seahorse Bioscience, North Billerica, MA, USA) with Seahorse XF medium supplemented with $10 \mathrm{mM}$ glucose, $1 \mathrm{mM}$ sodium pyruvate and $2 \mathrm{mM}$ glutamine. The plate was pretreated under a non- $\mathrm{CO}_{2}$ condition for $1 \mathrm{~h}$, sequentially injected with oligomycin (inhibition of ATP synthase), carbonyl cyanide-4-(trifluoromethoxy)phenylhydrazone, (uncoupling agent of oxygen consumption from ATP production) and rotenone and antimycin A (inhibitor of complex I and III in the electron transport chain of mitochondria) into each well, and then processed in the XF analyzer for the oxygen consumption rate (OCR) measurement and analysis. The compounds listed above were sequentially injected into each well to measure ATP production, maximal respiration and non-mitochondrial respiration.

\section{Data analysis \\ All the data are expressed as the mean \pm s.d. Student's $t$-test was applied for comparisons between two groups, and one-way analysis of variance followed by least significant difference $t$-test was used for comparisons of more than two different groups (GraphPad Prism software, GraphPad Software, San Diego, CA, USA). A $P$-value less than 0.05 was considered statistically significant.}

\section{RESULTS}

IL-25 correlated with body mass index and decreased in liver and eWAT

We examined the levels of IL-25 in the serum and liver biopsy samples from patients with or without NAFLD. IL-25 expression was decreased in the serum and liver of NAFLD patients who had a higher BMI, body mass index (BMI; Figures 1a and b). To further investigate the role of IL-25 in NAFLD, we established a diet-induced obesity (DIO) mouse model with hepatic steatosis. During the HFD treatment, the levels of IL-25 in the serum (Figure 1c) and liver (Figure 1d) were reduced from the second week of the HFD program. In the DIO animal model, both the mRNA and protein levels of IL-25 were reduced in the HFD-fed mice compared with those in the NCD-fed mice both in eWAT (Figure 1f) and liver (Figure 1e). We also observed the expression of IL-25 in the liver and eWAT of $\mathrm{DB} / \mathrm{DB}(\mathrm{Lepr}-/-)$ obese mice. The results were similar to those in the DIO mice; there was reduced expression of IL-25 in the liver and eWAT of DB/DB obese mice (Supplementary Figure S1). Therefore, IL-25 may correlate with obesity and associated metabolic diseases.

\section{IL-25 administration reduced body weight gain and lipid accumulation without affecting food intake via the alteration of metabolic enzymes in vivo}

To further investigate the metabolic role of IL-25 in lipogenesis or lipolysis during obesity, exogenous IL-25 was administered to HFD-fed obese mice at the end of the HFD-induction program. Mice fed with an NCD or an HFD for 3 months were administered BSA or IL-25 (1 $\mu \mathrm{g}$ in $100 \mu \mathrm{l}$ PBS for each mouse) every day for 7 days, followed by every other day for an additional 16 days for a total of 23 days. IL-25 decreased body weight gain and fat mass in the HFDfed mice (Supplementary Figures S2B and C) without affecting food intake (data not shown). The adipocyte size of eWAT was decreased in the HFD-fed mice treated with IL-25 (Figure 2d). IL-25 also decreased the liver mass of the HFD-fed mice (Supplementary Figure S2D), reduced lipid accumulation and alleviated hepatic steatosis in the liver (Figure 2g). Moreover, IL-25 promoted fat mobilization and lipolysis in the HFD-fed mice, with a decrease in TGs and an increase in FFA in the serum (Figure 2a), eWAT (Figure 2e) and liver (Figure 2h). In addition, the IL-25 administration increased the level of HDL, which is a protective lipoprotein against cardiovascular diseases, and decreased the level of LDL/VLDL, which is a risk factor for cardiovascular diseases, in the serum of the HFD-fed obese mice (Figure 2a). In eWAT and liver, fat mobilization was mediated by ATGL, which was decreased in the HFD-fed mice but increased during the treatment with IL-25 (Figures $2 \mathrm{~b}$ and $\mathrm{c}$ ). Although the mRNA of lipogenic enzymes, including Acly and Acaca, was not significantly varied, the mRNA of lipolytic enzymes such as Atgl, enzymes for long-chain acyl-CoA (or FFA) penetrating the membrane of mitochondria such as carnitine palmitoyltransferase-I $(C p t 1 \alpha)$, and enzymes for $\beta$-oxidation such as hydroxyacyl-coenzyme A dehydrogenase $(\mathrm{Hadh})$, were significantly upregulated during the treatment with IL-25 in eWAT (Figure 2f). Similar results were observed in the liver (Figure 2i). Consequently, the lipolytic effect of IL-25 may be associated with its regulation of some lipid metabolic enzymes.

\section{IL-25 may regulate lipid metabolism via alternatively activating macrophages in vivo and in vitro}

Macrophages have an important role not only in the innate immune responses but also in regulating metabolism homeostasis. Macrophages accumulating in adipose tissue and the liver can be polarized to different phenotypes that can secrete 

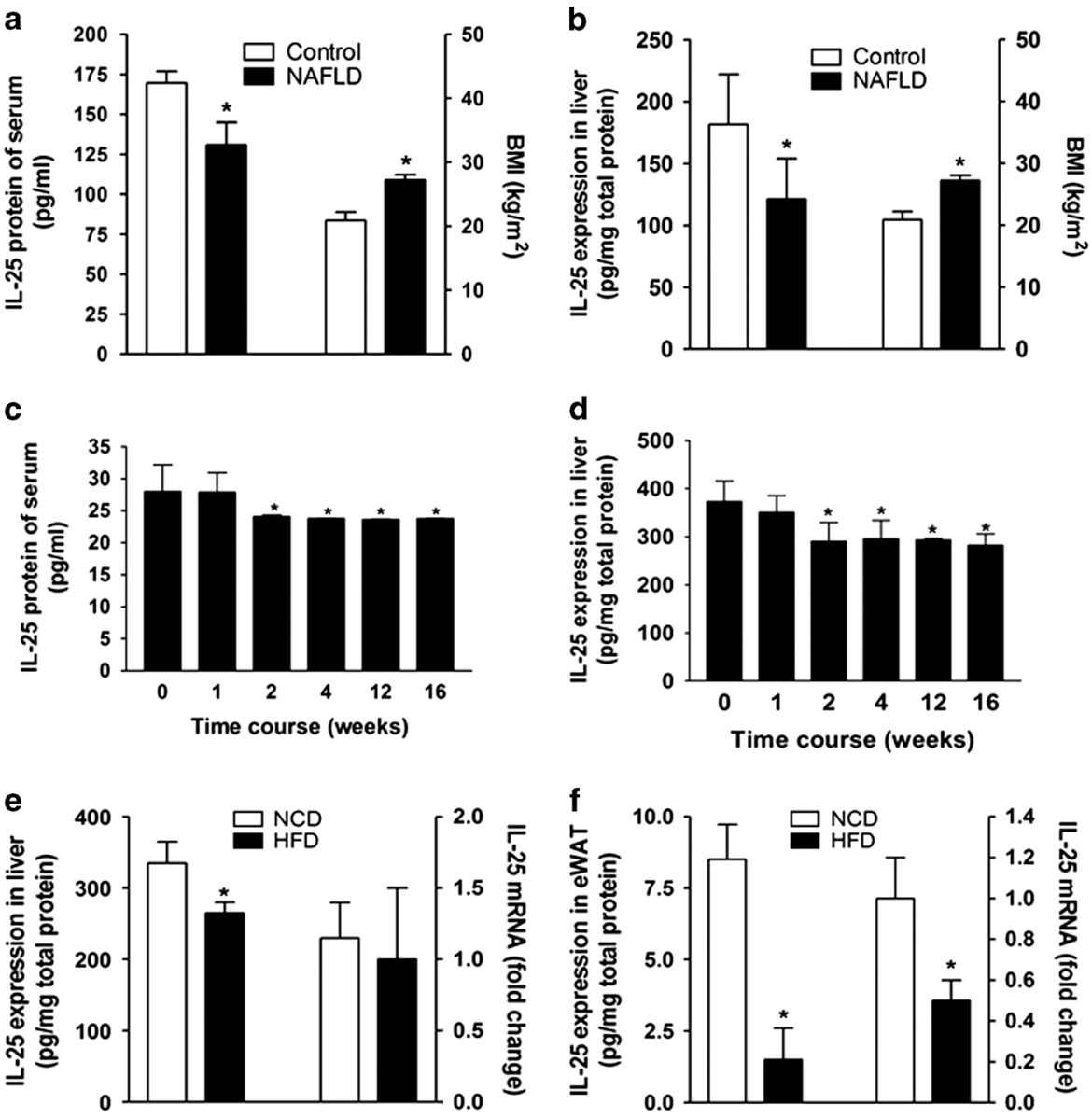

Figure $1 \mathrm{IL}-25$ correlates with BMI in NAFLD patients and is decreased in the eWAT and liver of diet-induced obese mice. (a, $\mathbf{b}$ ) The level of IL-25 in the serum (a) and liver (b) compared with the BMI of control and NAFLD patients. An ELISA was used to measure the level of IL-25. NAFLD $n=14$, control $n=8$; ${ }^{*} P<0.05$ versus the control group. (c, d) The expression of IL-25 in the serum (c) and liver (d) of mice fed with an HFD at different time points. An ELISA was used to measure the level of mouse IL-25. The data are presented as the mean \pm s.d. of three independent experiments. $n=5 ;{ }^{*} P<0.05$ versus the $0-\mathrm{W}$ group. (e, f) The expression of IL-25 in the livers (e) and eWAT (f) of mice fed with NCD/HFD. The level of IL-25 was measured by an ELISA and qPCR in the liver or eWAT. The data are presented as the mean \pm s.d. of three independent experiments. $n=5 ;{ }^{*} P<0.05$ versus the NCD group. BMI, body mass index; eWAT, epididymal white adipose tissue; HFD, high-fat diet; IL, interleukin; NAFLD, nonalcoholic fatty liver disease; NCD, normal chow diet; qPCR, quantitative real-time PCR.

various cytokines and have different functions depending on the microenvironment. With the immunofluorescent co-location of the macrophage marker F4/80 and the M2 phenotype marker ARG1, we found greater M2 macrophage accumulation in the eWAT (Figure 3a) and liver (Figure 3c) of IL-25-treated mice. We also examined the mRNA of some phenotype markers or cytokines in vivo and in vitro. Macrophages expressed more M2 markers (Arg1 and Ym1) and type2 immune cytokines (Il-13), but not M1 marker (Inos) and type-1 immune cytokines (Tnfo), in the eWAT (Figure 3b) and liver (Figure 3d) of HFD-fed mice during the treatment with IL-25. In BMMCs, macrophages also expressed more M2 markers (Arg1, Mgl1/2, Fizz and Ym1), M2-associated transcription factor (IRF4) and type-2 immune cytokines (Il-13, Il-5 and Il-4), but not the M1 marker (Inos), M1-associated transcription factor (IRF5) or type- 1 immune cytokines (Il-1 $\beta$ and Il-12b; Figures $3 e$ and f) after IL-25 treatment.
Furthermore, the expression levels of M2 markers (ARG1) and transcription factor (IRF4) were increased during the treatment with IL-25 in a concentration-dependent manner, whereas the expression of M1 transcription factor (IRF5) was not significantly varied (Supplementary Figures S3A-C). Therefore, IL-25 stimulated M2 polarization of macrophages both in vivo and in vitro. With the polarization of macrophages and changes in the cytokine profiles, the function and metabolic characteristics of macrophages were also switched.

\section{IL-25 increased lipid uptake and enhanced lipolysis via} macrophages

IL-25 participates in immune metabolism via regulating the phenotype and functions of macrophages. RAW264.7 macrophages and differentiated 3T3-L1 adipocytes were either directly stimulated with IL-25 and a type-1 cytokine (IFN $\gamma$ ) or a mediator (LPS) or were co-cultured together using a 
a

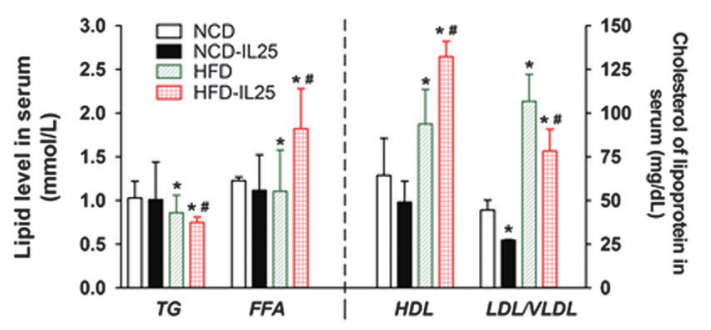

d

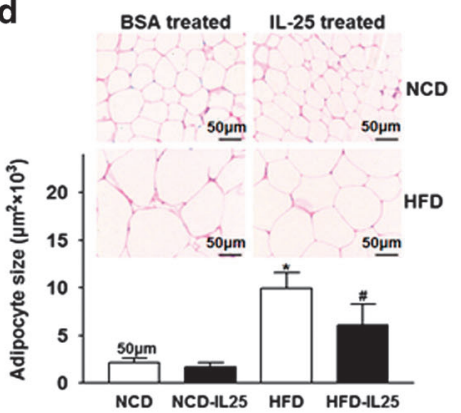

g

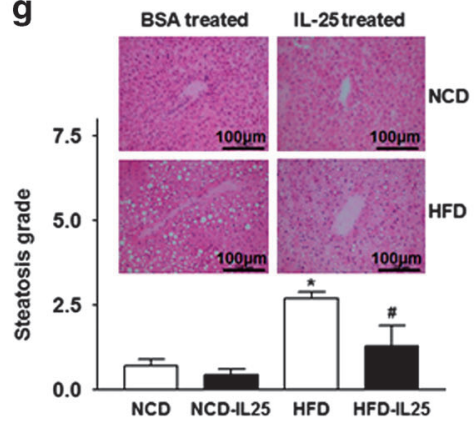

e

h b

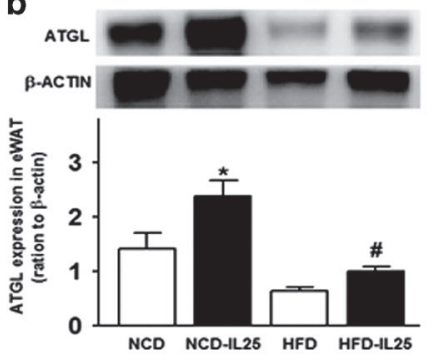

C

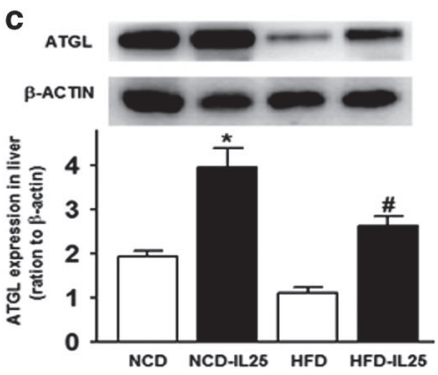

f
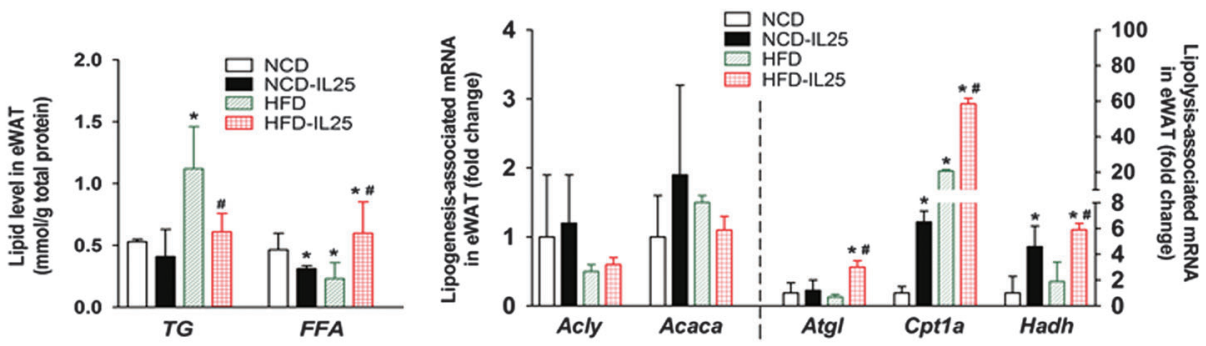

i
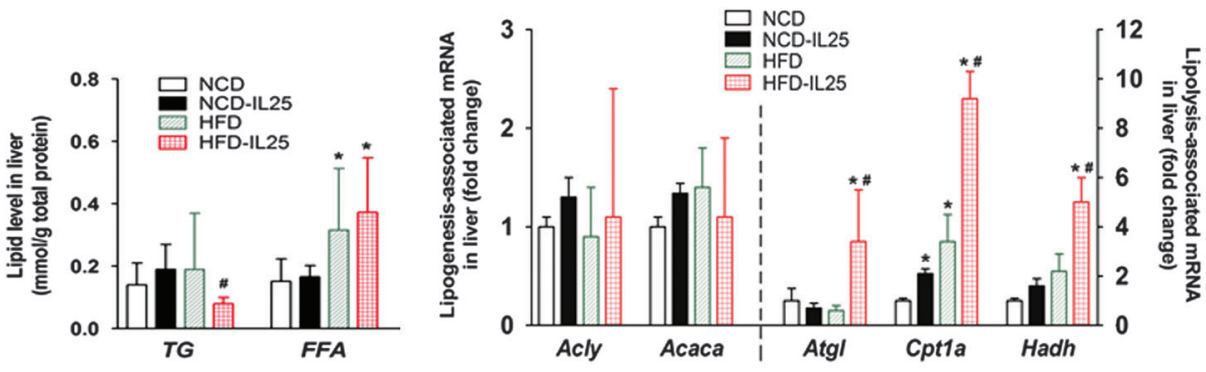

Figure 2 IL-25 promotes fat mobilization and reduces lipid accumulation via the alteration of lipid metabolic enzymes in vivo. Mice were fed with HFD or NCD for 3 months, re-grouped and then injected with BSA or IL-25 (1 $\mu$ g in $100 \mu$ l PBS for each mouse) every day for 7 days and then every other day for 16 days at the end of the HFD program. Real-time qPCR was applied to examine the gene expression of lipogenic enzymes, lipolytic enzymes and fatty-acid oxidation proteins in the eWAT or liver. The fold changes were relative to the BSAtreated group after normalization using the $18 \mathrm{~S}$ rRNA. The data are presented as the mean $\pm \mathrm{s}$.d. $n=5$; $P<0.05$ versus the NCD group, $\# P<0.05$ versus the HFD group. (a) The level of TG, FFA, HDL and LDL/NLDL in the serum. (b, c) The expression of ATGL in the eWAT (b) and liver (c). Gray scan histograms are presented as the mean \pm s.d. $n=5$; ${ }^{*} P<0.05$ versus the NCD group, ${ }^{\#} P<0.05$ versus the HFD group. (d, g) hematoxylin and eosin staining of the eWAT and liver, and the histogram of adipocyte size and steatosis grade. Adipocyte size was analyzed using the Image J software. Two to three images and 15-20 adipocytes per image were applied for the measurement. Slides were photographed at $\times 40$ magnification. $(\mathbf{e}, \mathbf{h})$ The level of TG and FFA in the eWAT (e) and liver (h) of mice. (f, i) The mRNA expression of lipogenesis and lipolysis associated genes in the eWAT (f) and liver (i). ATGL, adipose triglyceride lipase; BSA, bovine serum albumin; eWAT, epididymal white adipose tissue; FFA, free fatty acid; HFD, high-fat diet; IL, interleukin; NCD, normal chow diet; PBS, phosphatebuffered saline; qPCR, quantitative real-time PCR; TG, triglyceride.

0.4- $\mu \mathrm{m}$ Transwell chamber. During the co-culture, RAW264.7 macrophages were stimulated with IL-25, IFN $\gamma$ and LPS. As a pro-inflammatory cytokine and mediator, IFN $\gamma$ and LPS, respectively, were applied as an opposite control compared with IL-25. IL-25 contributed to lipid uptake (Figure 4a) and lipolysis via increasing the expression of lipolytic enzymes, such as p-HSL, ATGL and MAGL, whereas IFN $\gamma$ and LPS had little effects on lipolysis via decreasing p-HSL, ATGL and MAGL in macrophages (Figure 4b). In addition, IL-25 decreased the expression of lipogenic enzymes, such as fatty acid synthase (FAS), and inhibited the activity of ACC (a key enzyme in fatty-acid synthesis) by increasing its phosphorylation in macrophages (Figure 4b). Decreased lipid accumulation was observed in 3T3-L1 adipocytes cultured alone or co-cultured with RAW264.7 macrophages during the IL-25 treatment (Figure 4c). We further examined the metabolic enzymes in 3T3-L1 adipocytes stimulated with cytokines or co-cultured with cytokine-treated macrophages. The expression of lipolytic enzymes, such as p-HSL and ATGL, was increased in adipocytes co-cultured with IL-25-treated macrophages (Figure 4e), although there were little effects on adipocytes directly treated with IL-25 (Figure 4d). IL-25 also promoted lipid 
a

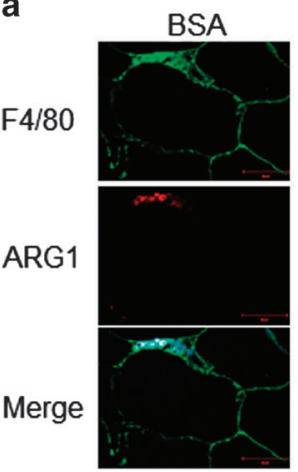

c
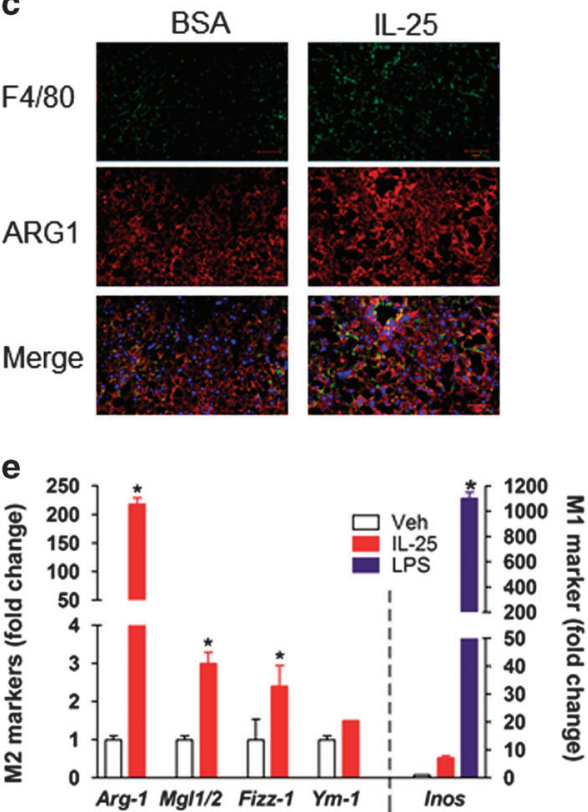

b

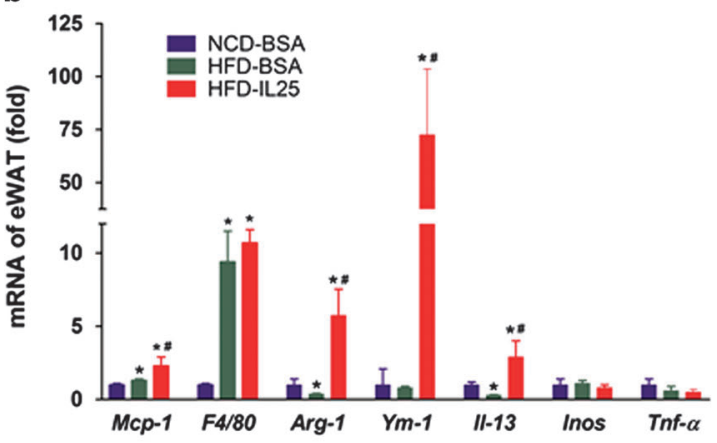

d
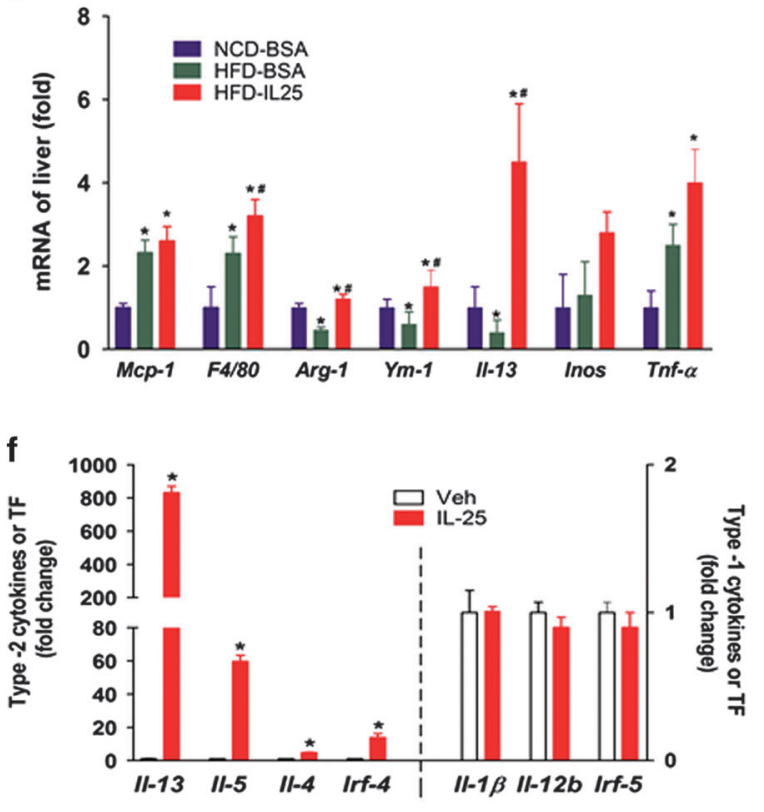

Figure 3 IL-25 alternatively activates macrophages (M2 polarization). (a, c) Immunofluorescent staining for F4/80 (macrophage marker) and ARG1 (M2 marker) in the eWAT (a) and liver (c). Images were representative of three independent experiments and photographed at $\times 40$ magnification. Scale bar: $50 \mu \mathrm{m}$. (b, d) The mRNA expression of the macrophage recruiting molecule Mcp-1, the macrophage marker F4/80, the M2 markers Arg-1 and Ym1, the type-2 immune cytokine II-13, the M1 marker Inos and the type-1 immune cytokine Tnfo in the eWAT (b) and liver (d) after IL-25 treatment. The data are presented as the mean \pm s.d. $n=5$; ${ }^{*} P<0.05$ versus the NCD group, ${ }^{\#} P<0.05$ versus the HFD group. (e, f) BMMCs were treated with IL-25 (50 ng/mL) for $72 \mathrm{~h}$. The expression of M1/M2 markers (e), type-1/type-2 cytokines and transcription factors (f) were examined in BMMCs. The data are presented as the mean \pm s.d. $n=3$; ${ }^{*} P<0.05$ versus the Veh group. BMMC, bone marrow-derived macrophage; eWAT, epididymal white adipose tissue; HFD, high-fat diet; IL, interleukin; NCD, normal chow diet.

uptake by macrophages and decreased lipid droplet accumulation in adipocytes in a concentration-dependent manner (Supplementary Figures S4A-C).

IL-25 increased the mitochondrial respiratory capacity as indicated by the increased expression of CPT1a, $\mathrm{NAD}^{+} / \mathrm{NADH}$ production, ATP production and OCR in macrophages

According to the theory of energy metabolism, mitochondrial respiratory capacity and energy metabolism are also crucial for the occurrence and development of obesity. As CPT1a is a key enzyme participating in $\beta$-oxidation of fatty acids in the mitochondria, we explored its expression in macrophages stimulated with cytokines. IL-25 increased the expression of CPT1a in macrophages (Figure 5a), which meant that IL-25 may transfer more fatty acids to the mitochondria for oxidation. As shown by the MitoTracker staining results, IL-25 amplified the mass of the mitochondria and promoted its infusion (Figure 5d), which indicated that IL-25 may improve mitochondrial respiratory capacity. IL-25 promoted total $\mathrm{NAD}^{+}$(NADt) or NADH production in macrophages (Figures $5 \mathrm{~b}$ and $\mathrm{c}$ ), which reflected greater potential energy via the respiratory chain. Finally, IL-25 increased the oxygen consumption rate (Figure 5f) and ATP production (Figure 5e) of the macrophages versus the control group, indicating that IL-25 may promote the aerobic oxidation and energy 
a

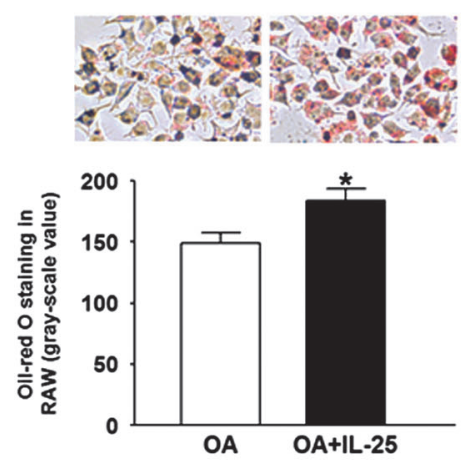

C
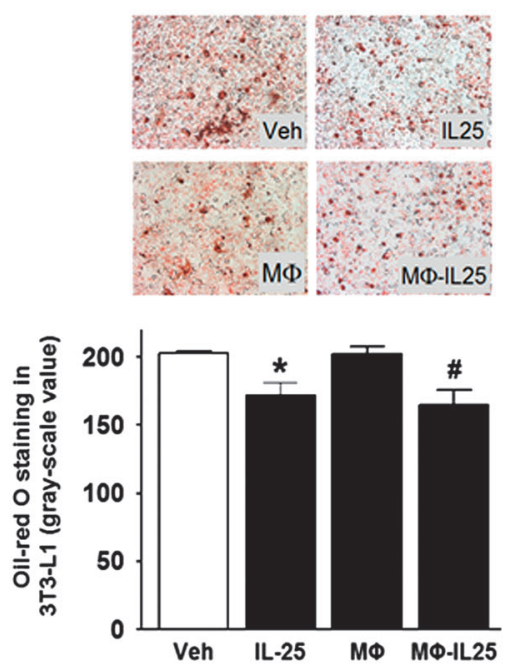

b Veh IL25 IFNy LPS

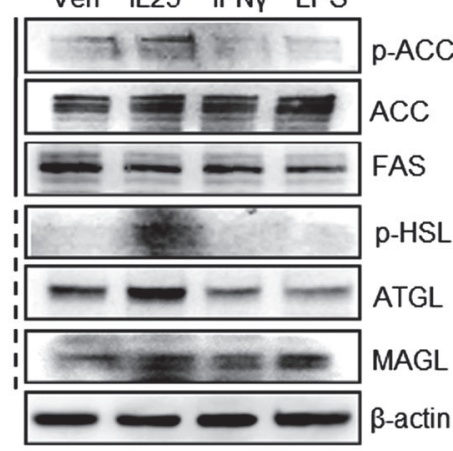

d

Veh IL-25 IFNy LPS
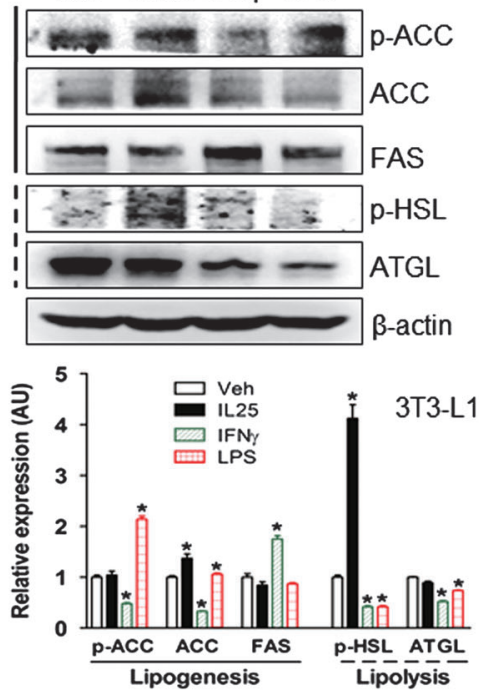

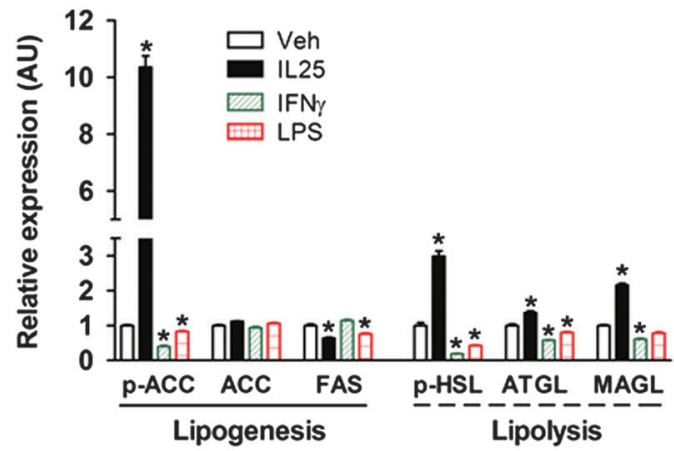

e
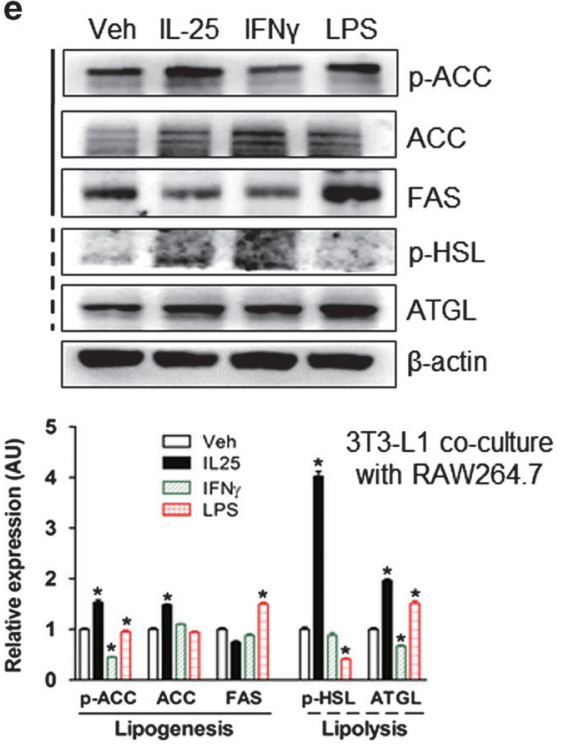

Figure 4 IL-25 enhances lipolysis in macrophages. (a) Lipid uptake in macrophages. Macrophages were able to take up more lipid droplets during treatment with IL-25 in the presence of OA (oleic acid). The ImageJ software was used for gray scanning. Gray histograms are presented as the mean \pm s.d. of three independent experiments. $n=5 ;{ }^{*} P<0.05$ versus the 0 A group. (b) The alteration of lipogenesis and lipolysis enzymes in RAW264.7 macrophages treated with cytokines. The left panel shows the typical western blot bands, and the right panel shows the quantitative analysis of the gray scan. The ImageJ software was used for gray scanning. ${ }^{*} P<0.05$ versus the Veh group. (c) Oil Red 0 staining of 3T3-L1 adipocytes treated with IL-25 or Mø-IL-25. The ImageJ software was used for gray scanning. The gray histograms are presented as the mean \pm s.d. of three independent experiments. $n=5$; ${ }^{*} P<0.05$ versus the Veh group, ${ }^{\#} P<0.05$ versus the $\mathrm{M} \varnothing$ group. (d, e) The alteration of the levels of lipolysis and lipogenesis enzymes in 3TL-L1 adipocytes treated with cytokines (d) or cocultured with RAW264.7 macrophages treated with cytokines (e). The gray histograms are presented as the mean \pm s.d. of three independent experiments. $n=5$; ${ }^{*} P<0.05$ versus the Veh group. Different cytokines such as IL-25 (50 ng/ml), IFN (50 ng/ml) and LPS $(10 \mathrm{ng} / \mathrm{ml})$ were applied. LPS, lipopolysaccharide.

production of macrophages. In summary, IL-25 promoted the oxidation of fatty acids by increasing the mitochondrial respiratory capacity.

Adoptive transfer of IL-25-educated macrophages into HFDfed mice decreased the lipid level and affected the mRNA expression of some enzymes associated with lipid metabolism

In consideration of the important role of macrophages in lipid metabolism, IL-25-educated macrophages (Mø-IL-25), MøIL-15 and Mø-IFN $\gamma$ were adoptively transferred to HFD-fed mice to explore the effects of IL-25 on lipid metabolism via macrophages. The adoptive transfer of Mø-IL-25 into HFD-fed mice decreased the lipid level in the serum (Figure 6a) and increased Atgl mRNA (Figure 6c), which had an important role in lipolysis. In addition, the adoptive transfer of Mø-IL-25 increased Cpt1a mRNA in the HFD-fed mice to promote lipid transportation to the mitochondria and $\beta$-oxidation to some extent (Figure 6e). Whereas the role of Mø-IL-15 and MøIFN $\gamma$ was not obvious in these above-mentioned aspects, MøIL-25, Mø-IL-15 and Mø-IFN $\gamma$ also decreased the expression of Cidea to promote lipolysis (Figure 6f), whereas the expression of $C d 36$, which participates in fatty-acid uptake (Figure $6 \mathrm{~d}$ ), was increased. Mø-IL-25 also increased the level of HDL and decreased the level of LDL/VLDL (Figure 6b), which may be helpful for cardiovascular diseases. 
a
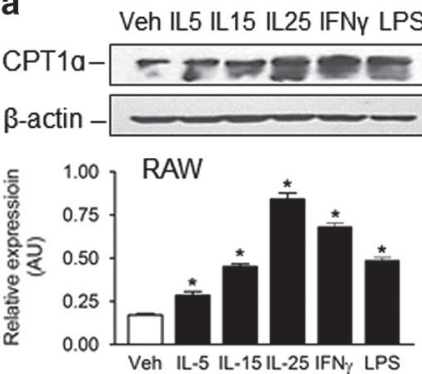

b

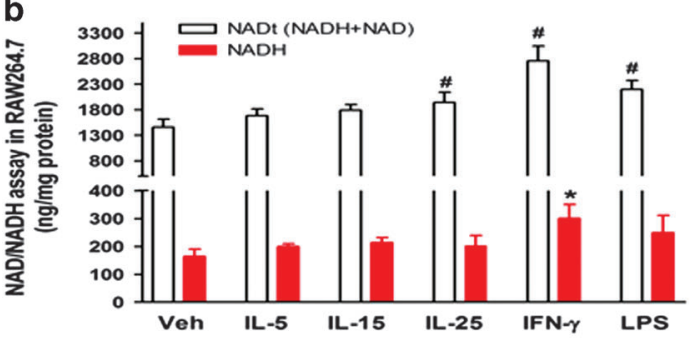

C

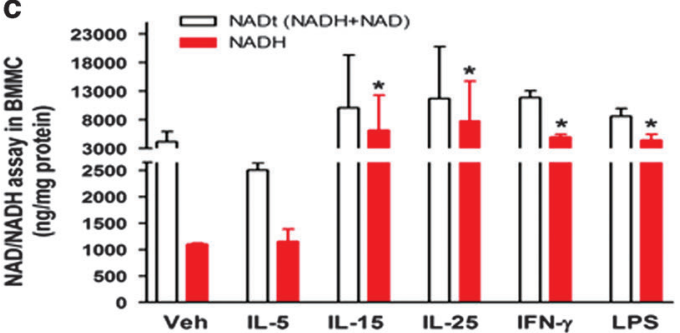

d
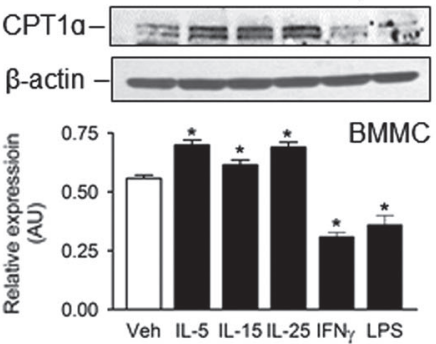
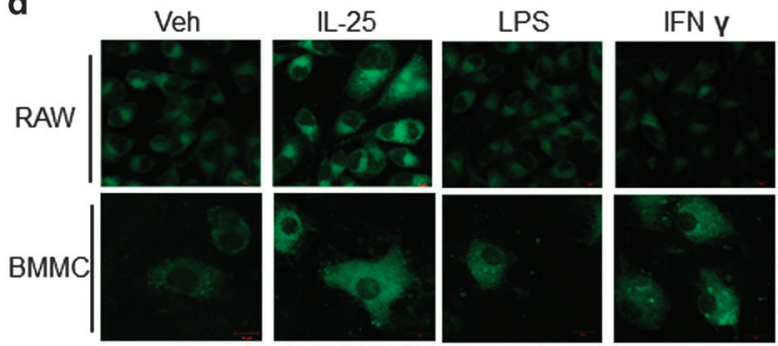

e
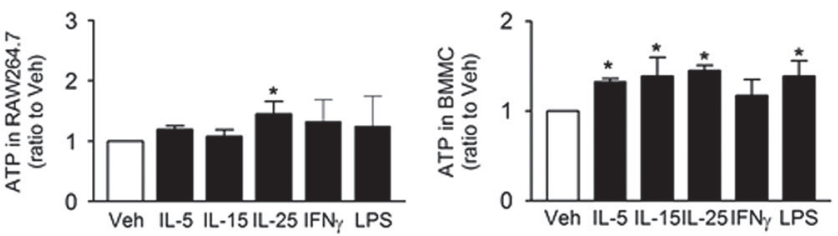

f

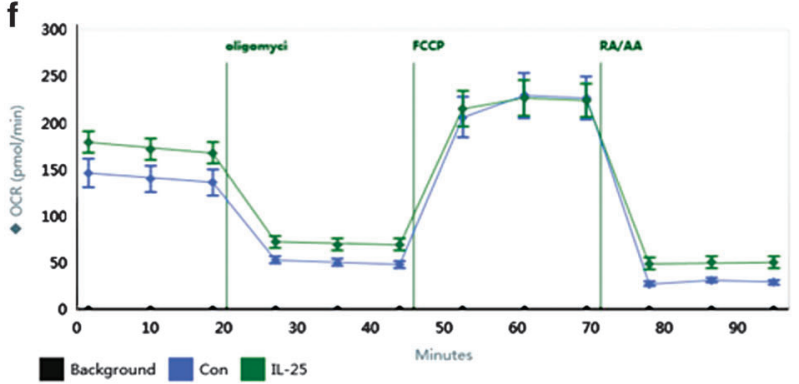

Figure 5 IL-25 increases the respiratory capacity of macrophages. RAW264.7 macrophages and/or BMMCs were treated for $48 \mathrm{~h}$ with different cytokines or mediators, such as IL-5, IL-15, IL-25, IFN $\gamma$ and LPS. (a) The expression of the CPT1a protein in RAW264.7 macrophages and BMMCs. (b, c) The total NAD (NADt) and decomposed NAD (NADH) production in RAW264.7 cells (b) and BMMCs (c) treated with cytokines. The data are presented as the mean \pm s.d. of three independent experiments. $n=3$; ${ }^{*} P<0.05$ versus the VehNADH group; ${ }^{\#} P<0.05$ versus the Veh-NADt group. (d) MitoTracker staining showed the mitochondrial mass and fusion in RAW264.7 macrophages and BMMCs treated with cytokines. Scale bar: $10 \mu \mathrm{m}$. (e) The ATP production in RAW264.7 cells and BMMCs treated with cytokines. The data are presented as the mean \pm s.d. of three independent experiments. $n=3 ;{ }^{*} P<0.05$ versus the Veh group. (f) OCRs in BMMCs. The data are presented as the mean \pm s.d., $n=5$. BMMC, bone marrow-derived macrophage; IFN, interferon; IL, interleukin; LPS, lipopolysaccharide; OCR, oxygen consumption rate.

\section{DISCUSSION}

This study showed that IL-25 expression was reduced in NAFLD patients and a mouse model of obesity with greater lipid accumulation. The results indicate that IL-25 may be associated with obesity and lipid metabolism. We further found that the administration of exogenous IL-25 reduced body weight gain and the mass of both eWAT and liver in HFDfed mice. Both the adipocyte size of eWAT and lipid accumulation in the liver were reduced in the HFD-fed mice after the IL-25 treatment. The results indicated that IL-25 promoted lipid metabolism. The protective effect of IL-25 on hepatic steatosis was in accordance with the results previously reported. ${ }^{21}$ As macrophages accumulate in adipose tissue, we further explored the potential role of IL-25-triggered and macrophage-mediated immunometabolism in this system.

IL-25 is a member of the IL-17 family, which participates in anti-inflammatory responses. ${ }^{32}$ IL-25 targets macrophages to induce type- 2 immunity. ${ }^{24}$ In our study, we identified that IL-25-stimulated macrophages expressed more M2 markers and cytokines in vitro. In vivo, there were more ARG1 and F4/80 double-positive cells accumulated in both the liver and eWAT of IL-25-treated HFD-fed mice. Therefore, IL-25 promoted the M2 polarization of macrophages. M2 macrophages may prefer the $\beta$-oxidation of fatty acids as fuel to produce energy and metabolites. ${ }^{33,34}$ Our results indicated that IL-25 promoted lipid metabolism via M2 polarization of macrophages.

In our study, we further explored the metabolic characteristics of macrophages stimulated with IL-25. In macrophages, IL-25 enhanced the expression of lipolytic enzymes (ATGL, MAGL and p-HSL), decreased the expression of the lipogenic enzyme FAS and inhibited the activity of the lipogenic enzyme ACC by increasing its phosphorylation as the phosphorylated form of ACC is inactive. It is known that ATGL and HSL are 

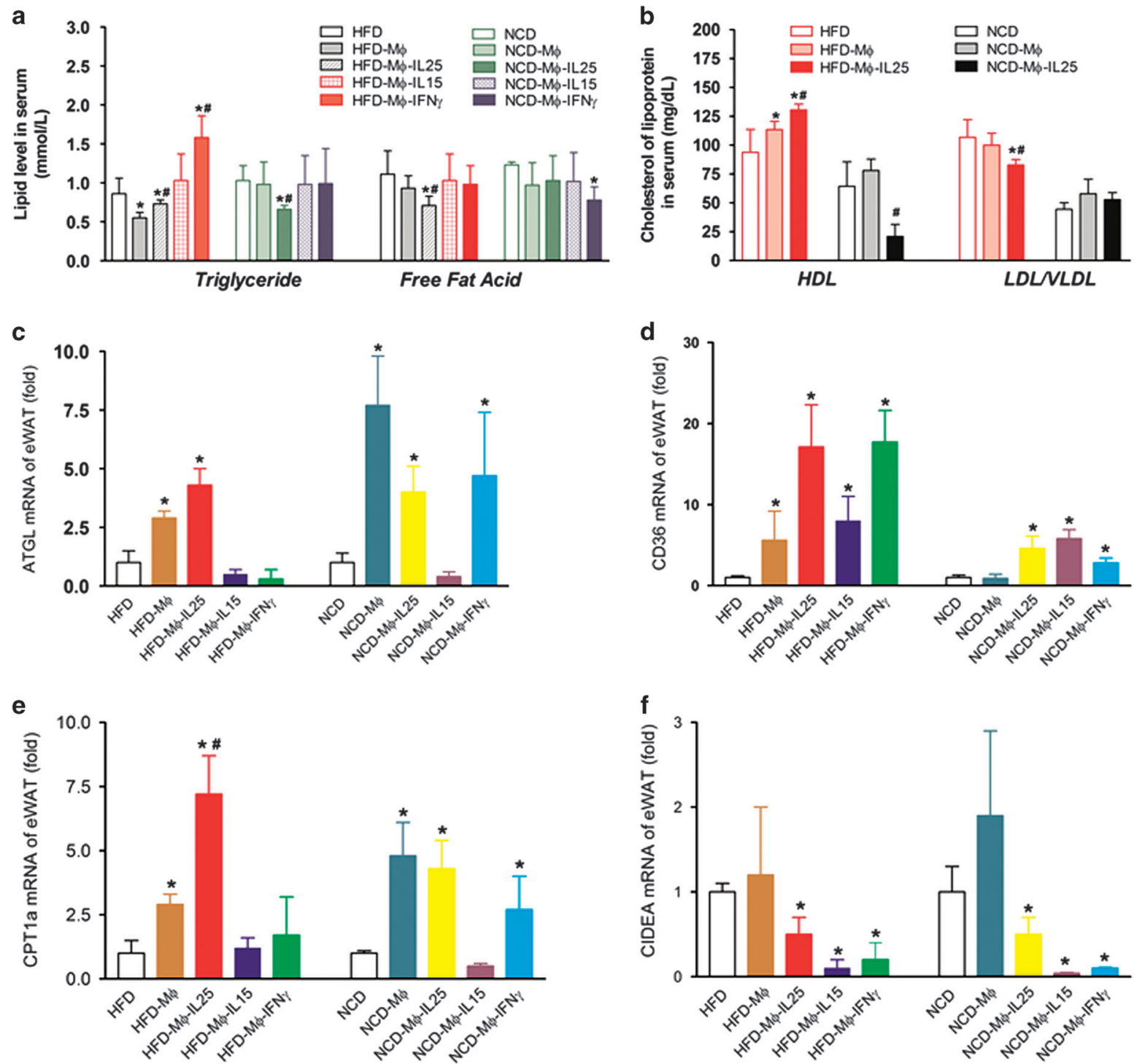

Figure 6 Mø-IL-25 promotes the expression of enzymes responsible for lipolysis and the translocation and oxidation of lipids. For adoptive transfer, BMMCs stimulated with or without IL-25, IL-15 and IFN $\gamma$ for 2 days were collected in cold PBS, and $1 \times 10^{6}$ cells were adoptively transferred into every mouse intraperitoneally at the end of the HFD program. The serum and eWAT tissues were collected to perform the measurements 2 days after the adoptive transfer of activated macrophages. The data are presented as the mean $\pm \mathrm{s}$. $\mathrm{d}$. $\mathrm{n}=5$; ${ }^{*} P<0.05$ versus the NCD or HFD group; ${ }^{\#} P<0.05$ versus the HFD-M $\varnothing$ group. (a) The lipid level in the serum after the adoptive transfer of Mø-IL-25, Mø-IL-15 and Mø-IFN $\gamma$. (b) The level of HDL and LDL/NLDL lipoproteins in the serum after adoptive transfer. (c) The mRNA expression of Atgl in the eWAT. (d) The mRNA expression of Cd36 in the eWAT. (e) The mRNA expression of Cpt1a in the eWAT. (f) The mRNA expression of Cidea in the eWAT. BMMC, bone marrow-derived macrophage; eWAT, epididymal white adipose tissue; HFD, high-fat diet; IFN, interferon; IL, interleukin; NCD, normal chow diet; PBS, phosphate-buffered saline.

the rate-limiting enzymes that hydrolyze stored esters to FFAs. ${ }^{35,36}$ ATGL, which is expressed predominantly in adipose tissue, is also found to hydrolyze intracellular TGs into FFAs within cardiac and skeletal muscle. ${ }^{35} \mathrm{HSL}$, which is expressed not only in adipose tissue but also in steroidogenesis tissue such as testis, hydrolyzes stored TGs or cholesteryl esters to FFAs. ${ }^{36,37}$ FAS is a multienzyme that catalyzes fatty-acid synthesis, and ACC is the rate-limiting enzyme participating in the first step of fatty-acid synthesis. Consequently, the more lipids that macrophages engulf, the more lipolysis that occurs under an IL-25 challenge. After releasing more FFAs, IL-25 significantly promoted the transportation and oxidation of FFA. It was interesting that the mRNA expression of lipolytic enzyme genes such as Cptla, Hadh and Atgl increased, but expression did not increase for the lipogenic enzyme genes, such as Acly and Acaca. CPT1a is responsible for transferring long-chain fatty acids into the mitochondria for $\beta$-oxidation. With the regulation of lipid metabolism enzymes, the lipolysis ability of macrophages was enhanced. Moreover, we observed that IL-25 amplified the mitochondrial capacity and promoted mitochondrial infusion, as shown by the MitoTracker staining. It indicated that IL-25 may influence $\beta$-oxidation and energy 
production. We further observed that IL-25 increased OCR, $\mathrm{ATP}$ and $\mathrm{NAD}^{+} / \mathrm{NADH}$ production in vitro, although we also observed that type-1 cytokines such as IL-15 promote the expression of CPTla and increase ATP and $\mathrm{NAD}^{+} / \mathrm{NADH}$ production, which is consistent with a previous report that IL-15 can regulate spare respiratory capacity and oxidative metabolism by promoting mitochondrial biogenesis and expression of CPT1a. ${ }^{38}$ IL-15 was also reported to induce weight loss and improve glucose homeostasis in obese mice. ${ }^{39}$ Therefore, cytokines, including IL-25 and IL-15, may have an important role in obesity by influencing lipid or glucose metabolism. It has been reported that M1 macrophages tend to utilize the glycolytic pathway for energy generation, whereas M2 macrophages may prefer the $\beta$-oxidation of fatty acids as fuel. Thus, we also examined the role of two other type-1 cytokines, IFN $\gamma$ and LPS, on the production of NADt/NADH and ATP as the type-1 cytokine control. Even though IL-15, IFN $\gamma$ and LPS also increased the production of NADt/NADH and ATP to some extent compared with the Veh group, which seems similar to IL-25, the mechanism of these cytokines may be different. The type- 1 cytokines, such as IFN $\gamma$ and LPS, may utilize the glycolytic pathway, whereas IL-25 may prefer $\beta$-oxidation of fatty acids as fuel. Our results (such as Figures $6 \mathrm{a}$ and $\mathrm{c}$ ) also indicate that the effect of IL-25 on lipolysis seems to be more obvious than the effect caused by type-1 cytokines such as IL-15 and IFN $\gamma$. IL-5 is mainly produced by Th2 lymphocytes participating in type- 2 immune responses and regulating AAM. We also used it as a type-2 immune response control to compare with IL-25.

We explored the effects of IL-25 and Mø-IL-25 on adipocytes and lipid metabolism. Both IL-25 and Mø-IL-25 diminished lipid accumulation in 3T3-L1 adipocytes. This may be associated with the increased expression of lipolytic enzymes (ATGL, MAGL and p-HSL) and the decreased expression of lipogenic enzymes (FAS) in macrophages or 3T3-L1 adipocytes treated with IL-25 or Mø-IL-25. These results suggested that IL-25 influences the metabolic characteristics of macrophages and adipocytes. IFN $\gamma$ and LPS were used as type-1 immune response controls, and they are typical type-1 cytokines or mediators for the classical activation of macrophages. It seems that the expression of p-HSL, ATGL and MAGL increased in 3T3-L1 adipocytes co-cultured with macrophages stimulated with IL-25 or IFN $\gamma$ compared with the Veh group, whereas IL-25 largely increased the p-ACC level compared with the Veh group and the groups treated with the other two cytokines (IFN $\gamma$ and LPS; Figure 4e). In macrophages, IL-25 increased the levels of p-ACC, ATGL and p-HSL more (Figure 4b) than that in the Veh group and the groups treated with the other two cytokines. Thus, we should consider the integrated effects of IL-25 on lipolysis in many aspects altogether, such as the effects of IL-25 on macrophages, adipocytes and their interaction. It has been reported that IL-33, another type-2 cytokine, also diminished lipid accumulation and delayed adipocyte differentiation. ${ }^{40}$ Furthermore, the adoptive transfer of

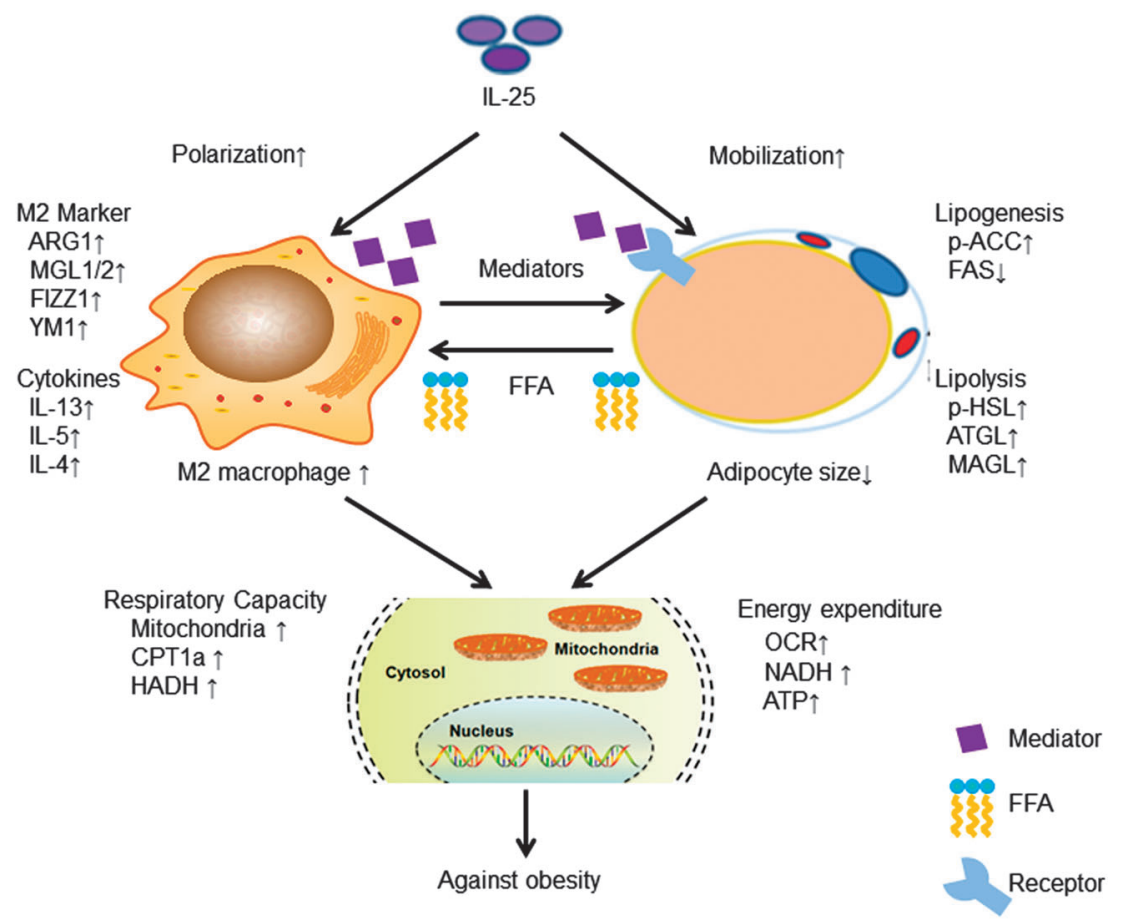

Figure 7 A proposed model for the immunometabolism roles and functions of IL-25 related to macrophages and adipocytes. This schema summarizes the main roles and functions of IL-25 in lipid metabolism. IL-25 can polarize macrophages into the M2 phenotype and secrete type-2 cytokines/mediators to promote fat mobilization, release FFA, enhance the lipid uptake by macrophages, improve the mitochondrial respiratory capacity and promote energy expenditure against obesity. FFA, free fatty acid; IL, interleukin. 
Mø-IL-25 into HFD-fed mice also had similar effects, such as decreasing the level of serum lipids, increasing the expression of Atgl and Cpt1a, the hydrolysis of TG and the transportation of FFA to the mitochondria for $\beta$-oxidation.

It seems paradoxical to compare the level of FFA between HFD-IL-25 treatment and the adoptive transfer of HFDMø-IL-25. However, they are two different experiments that have the following two major differences: one focuses on the uptake of FFA, and the other addresses the transportation of FFA across the mitochondrial membrane for $\beta$-oxidation. Our data demonstrated that the adoptive transfer of macrophages treated by IL-25 resulted in the macrophages engulfing more FFA (Figure 4a and Supplementary Figure S4). The adoptive transfer also enhanced the level of CPT1 $\alpha$ to a level greater than that achieved by the IL-25 injection as the IL-25 injection directly increased CPT1 $\alpha$ by approximately twofold (Figure $2 \mathrm{f}$ ). The adoptive transfer of macrophages treated with IL-25 increased the level of CPT $1 \alpha$ by approximately sevenfold (Figure 6f). Consequently, IL-25 may regulate enzymes related to the promotion of lipolysis and the inhibition of lipogenesis both in macrophages and adipocytes.

In conclusion, IL-25 promotes lipid metabolism and energy production, improves mitochondrial respiratory capacity and alleviates lipid accumulation in the liver and adipose tissues via alternatively activated macrophages and its interaction with adipocytes (Figure 7). Moreover, the administration of IL-25 reduces obese body weight gain, alleviates hepatic steatosis and regulates enzymes involved in lipid metabolism. The role of IL-25 in immunometabolism may contribute to ameliorating metabolic dysfunction in obesity.

\section{CONFLICT OF INTEREST}

The authors declare no conflict of interest.

\section{ACKNOWLEDGEMENTS}

This study was supported by the National Nature Science Foundation of China: 81272338, 81272515, 81370945, 81471033, 81570871, 81570764 and 81600641; the National Key Sci-Tech Special Project of China: 2013ZX09102-053, 2015GKS-355; the Program for Doctoral Station in University: 20130171110053; the Key Project of the Nature Science Foundation of Guangdong Province, China: 2015A030311043, 2016A030311035; the Guangdong Natural Science Fund: 2014A020 212023, 2014A030313073, 2015A030313103 and 2015A030313029; the Guandong Science and Technology Project (2014A020212023, 2015B0 90903063 and 2016A020214001); the Guangzhou Science and Technology Project: 2014J4100162, 201508020033 and 201510010052; the Pearl River Nova Program of Guangzhou Municipality, China, Grant Number: 201610010186; the Chang jiang Scholars and Innovative Research Team in University: 985 project PCSIRT 0947; and the Fundamental Research Funds for the Central Universities of China (Youth Program 13ykpy06, 16ykpy24 and 31610046).

\section{DISCLAIMER}

The funding agencies had no role in the study design, data collection and analysis, decision to publish or preparation of the manuscript.
1 Castoldi A, Naffah de Souza C, Camara NO, Moraes-Vieira PM. The macrophage switch in obesity development. Front Immunol 2015; 6: 637.

2 WHO. World Health Organization. Available from http://www.who.int/ mediacentre/factsheets/fs311/.

3 Woo Baidal JA, Lavine JE. The intersection of nonalcoholic fatty liver disease and obesity. Sci Trans/ Med 2016; 8: 323rv321.

4 Poloz Y, Stambolic V. Obesity and cancer, a case for insulin signaling. Cell Death Dis 2015; 6: e2037.

5 Kim SH, Despres JP, Koh KK. Obesity and cardiovascular disease: friend or foe? Eur Heart J 2015; epub ahead of print 18 December 2015; doi:10.1093/eurheartj/ehv509.

6 Moley KH, Colditz GA. Effects of obesity on hormonally driven cancer in women. Sci Trans/ Med 2016; 8: 323ps323.

7 Baffi CW, Wood L, Winnica D, Strollo Jr PJ, Gladwin MT, Que LG et al. Metabolic syndrome and the lung. Chest 2016; 149: 1525-1534.

8 Cani PD, Amar J, Iglesias MA, Poggi M, Knauf C, Bastelica D et al. Metabolic endotoxemia initiates obesity and insulin resistance. Diabetes 2007; 56: 1761-1772.

9 Schaffler A, Scholmerich J. Innate immunity and adipose tissue biology. Trends Imunol 2010; 31: 228-235.

10 Ahima RS, Flier JS. Adipose tissue as an endocrine organ. Trends Endocrinol Metab 2000; 11: 327-332.

11 Ahima RS. Adipose tissue as an endocrine organ. Obesity 2006; 14: 242S-249S.

12 Dalmas E, Clement K, Guerre-Millo M. Defining macrophage phenotype and function in adipose tissue. Trends Imunol 2011; 32: 307-314.

13 Tordjman J, Poitou C, Hugol D, Bouillot JL, Basdevant A, Bedossa P et al. Association between omental adipose tissue macrophages and liver histopathology in morbid obesity: influence of glycemic status. J Hepatol 2009; 51: 354-362.

14 Lumeng CN, Bodzin JL, Saltiel AR. Obesity induces a phenotypic switch in adipose tissue macrophage polarization. J Clin Invest 2007; 117: 175-184.

15 Eljaafari A, Robert M, Chehimi M, Chanon S, Durand C, Vial G et al. Adipose tissue-derived stem cells from obese subjects contribute to inflammation and reduced insulin response in adipocytes through differential regulation of the Th1/Th17 balance and monocyte activation. Diabetes 2015; 64: 2477-2488.

16 Ahmed M, Gaffen SL. IL-17 inhibits adipogenesis in part via C/EBP $\alpha$, PPAR $\gamma$ and Krüppel-like factors. Cytokine 2013; 61: 898-905.

17 Song X, He X, Li X, Qian Y. The roles and functional mechanisms of interleukin-17 family cytokines in mucosal immunity. Cell Mol Immunol 2016; 13: 418-431.

18 Kolls JK, Linden A. Interleukin-17 family members and inflammation. Immunity 2004; 21: 467-476.

19 Moseley TA, Haudenschild DR, Rose L, Reddi AH. Interleukin-17 family and IL-17 receptors. Cytokine Growth Factor Rev 2003; 14: 155-174.

20 Owyang AM, Zaph C, Wilson EH, Guild KJ, McClanahan T, Miller HR et al. Interleukin 25 regulates type 2 cytokine-dependent immunity and limits chronic inflammation in the gastrointestinal tract. J Exp Med 2006; 203: 843-849.

21 Wang AJ, Yang Z, Grinchuk V, Smith A, Qin B, Lu N et al. IL-25 or IL-17E protects against high-fat diet-induced hepatic steatosis in mice dependent upon IL-13 activation of STAT6. J Immunol 2015; 195: 4771-4780.

22 Sarra M, Cupi ML, Bernardini R, Ronchetti G, Monteleone I, Ranalli M et al. IL-25 prevents and cures fulminant hepatitis in mice through a myeloid-derived suppressor cell-dependent mechanism. Hepatology 2013; 58: 1436-1450.

23 Hams E, Locksley RM, McKenzie AN, Fallon PG. Cutting edge: IL-25 elicits innate lymphoid type 2 and type II NKT cells that regulate obesity in mice. J Immunol 2013; 191: 5349-5353.

24 Yang Z, Grinchuk V, Urban JF Jr., Bohl J, Sun R, Notari L et al. Macrophages as IL-25/IL-33-responsive cells play an important role in the induction of type 2 immunity. PLOS One 2013; 8: e59441.

25 Dai Z, Qi W, Li C, Lu J, Mao Y, Yao Y et al. Dual regulation of adipose triglyceride lipase by pigment epithelium-derived factor: a novel mechanistic insight into progressive obesity. Mol Cell Endocrinol 2013; 377: 123-134. 
26 Yang Z, Grinchuk V, Smith A, Qin B, Bohl JA, Sun R et al. Parasitic nematode-induced modulation of body weight and associated metabolic dysfunction in mouse models of obesity. Infect Immunity 2013; 81: 1905-1914.

27 Chalasani N, Younossi Z, Lavine JE, Diehl AM, Brunt EM, Cusi K et al. The diagnosis and management of non-alcoholic fatty liver disease: Practice Guideline by the American Gastroenterological Association, American Association for the Study of Liver Diseases, and American College of Gastroenterology. Gastroenterology 2012; 142: 1592-1609.

28 Brestoff JR, Kim BS, Saenz SA, Stine RR, Monticelli LA, Sonnenberg GF et al. Group 2 innate lymphoid cells promote beiging of white adipose tissue and limit obesity. Nature 2015; 519: 242-246.

29 Dranka BP, Benavides GA, Diers AR, Giordano S, Zelickson BR, Reily $C$ et al. Assessing bioenergetic function in response to oxidative stress by metabolic profiling. Free Radic Biol Med 2011; 51: 1621-1635.

30 Schneider L, Giordano S, Zelickson BR, S Johnson M, A Benavides G, Ouyang $\mathrm{X}$ et al. Differentiation of SH-SY5Y cells to a neuronal phenotype changes cellular bioenergetics and the response to oxidative stress. Free Radic Biol Med 2011; 51: 2007-2017.

31 Cooper O, Seo H, Andrabi S, Guardia-Laguarta C, Graziotto J, Sundberg $\mathrm{M}$ et al. Pharmacological rescue of mitochondrial deficits in iPSC-derived neural cells from patients with familial Parkinson's disease. Sci Trans/ Med 2012; 4: 141ra190.

32 Beringer A, Noack M, Miossec P. IL-17 in chronic inflammation: from discovery to targeting. Trends Mol Med 2016; 22: 230-241.

33 Johnson AR, Milner JJ, Makowski L. The inflammation highway: metabolism accelerates inflammatory traffic in obesity. Immunol Rev 2012; 249: 218-238.

34 Vats D, Mukundan L, Odegaard JI, Zhang L, Smith KL, Morel CR et al. Oxidative metabolism and PGC-1 beta attenuate macrophage-mediated inflammation. Cell Metab 2006; 4: 13-24.

35 Zimmermann R, Strauss JG, Haemmerle G, Schoiswohl G, Birner-Gruenberger $\mathrm{R}$, Riederer $\mathrm{M}$ et al. Fat mobilization in adipose tissue is promoted by adipose triglyceride lipase. Science 2004; 306 : 1383-1386.

36 Mulder H, Holst LS, Svensson H, Degerman E, Sundler F, Ahrén B et al. Hormone-sensitive lipase, the rate-limiting enzyme in triglyceride hydrolysis, is expressed and active in beta-cells. Diabetes 1999; 48: 228-232.

37 Kraemer FB, Shen WJ. Hormone-sensitive lipase: control of intracellular tri-(di-)acylglycerol and cholesteryl ester hydrolysis. J Lipid Res 2002; 43: 1585-1594.

38 van der Windt GJ, Everts B, Chang CH, Curtis JD, Freitas TC, Amiel E et al. Mitochondrial respiratory capacity is a critical regulator of CD8+ T cell memory development. Immunity 2012; 36: 68-78.

39 Sun H, Ma Y, Gao M, Liu D. IL-15/sIL-15R $\alpha$ gene transfer induces weight loss and improves glucose homeostasis in obese mice. Gene Ther 2016; 23: 349-356.

40 Martinez-Martinez E, Cachofeiro V, Rousseau E, Álvarez V, Calvier L, Fernández-Celis A et al. Interleukin-33/ST2 system attenuates aldosterone-induced adipogenesis and inflammation. Mol Cell Endocrinol 2015; 411: 20-27.

(i) (2) (2) This work is licensed under a Creative Commons Attribution-NonCommercial-ShareAlike $\quad 4.0$

International License. The images or other third party material in this article are included in the article's Creative Commons license, unless indicated otherwise in the credit line; if the material is not included under the Creative Commons license, users will need to obtain permission from the license holder to reproduce the material. To view a copy of this license, visit http://creativecommons.org/licenses/by-nc-sa/4.0/

C) The Author(s) 2018

Supplementary Information for this article can be found on the Cellular \& Molecular Immunology website (http://www.nature.com/cmi) 